\title{
TRATAMENTOS DE PRÉ-RESFRIAMENTO E RESFRIAMENTO SOBRE A QUALIDADE DE CARNE DE PEITO DE FRANGO'
}

\author{
Maria Cristina BRESSAN ${ }^{2, *}$, Nelson José BERAQUET ${ }^{3}$
}

\section{RESUMO}

O total de 402 frangos foi processado em abatedouro comercial e submetido a seis tratamentos de resfriamento. Inicialmente as carcaças foram pré-resfriadas (PR) por imersão em água e gelo, seguido de resfriamento (R) a - $35^{\circ} \mathrm{C}$ e estocagem a $4^{\circ} \mathrm{C}$ por 20 horas. Os tratamentos foram: A $\left(0^{\circ} \mathrm{C} / 30 \mathrm{~min},-35^{\circ} \mathrm{C} / 3 \mathrm{~h}\right.$ e $\left.15 \mathrm{~min}\right), \mathrm{B}\left(10^{\circ} \mathrm{C} / 30 \mathrm{~min}, 0^{\circ} \mathrm{C} / 30 \mathrm{~min},-35^{\circ} \mathrm{C} / 2 \mathrm{~h} \mathrm{e} 45 \mathrm{~min}\right), \mathrm{C}\left(10^{\circ} \mathrm{C} / 30 \mathrm{~min},-35^{\circ} \mathrm{C} / 3 \mathrm{~h}\right.$ e $15 \mathrm{~min}), \mathrm{D}\left(20^{\circ} \mathrm{C} / 30 \mathrm{~min}, 0^{\circ} \mathrm{C} / 30 \mathrm{~min},-35^{\circ} \mathrm{C} / 2 \mathrm{~h}\right.$ e $\left.45 \mathrm{~min}\right), \mathrm{E}\left(20^{\circ} \mathrm{C} / 30 \mathrm{~min},-35^{\circ} \mathrm{C} / 3 \mathrm{~h}\right.$ e $\left.15 \mathrm{~min}\right)$ e $\mathrm{F}\left(20^{\circ} \mathrm{C} / 30 \mathrm{~min}, 0^{\circ} \mathrm{C} / 3 \mathrm{~h}\right.$ e $\left.15 \mathrm{~min}\right) . \mathrm{Temp}^{-}$ raturas baixas utilizadas após a evisceração aceleraram a instalação do rigor em músculos pectoralis major (PM). Aos 45min post mortem carcaças sem PR (A) ou PR a $10^{\circ} \mathrm{C}$ (B) tiveram músculo PM com menor (P<0,001) pH $(5,75$ e 5,81$)$ do que carcaças $\mathrm{PR}$ a $20^{\circ} \mathrm{C}$ (D) $(5,95)$. Às 4 h p.m, nos tratamentos A e B as médias de valor $\mathrm{R}^{*}$ foram $(\mathrm{P}<0,05)$ mais elevadas $(1,51$ e 1,44$)$ que o no tratamento $\mathrm{D}(1,32)$. O teor de luminosidade foi influenciado $(\mathrm{P}<0,001)$ pelas temperaturas de $\mathrm{R}$ (nos tratamentos $\mathrm{A}$, B e $\mathrm{C}$ as médias foram de 48,2; 47,7 e 47,6 e nos tratamentos D e E de 45,5 e 45,7, respectivamente). Os teores de luminosidade mais elevados coincidiram com tratamentos com rápida glicólise post mortem. A perda de peso por cozimento e a força de cisalhamento não revelaram efeito dos tratamentos.

* razão entre as absorbâncias de 250nm e 260nm, que avalia a quantidade de monofosfato de inosina (IMP) para trifosfato de adenosina (ATP) Palavras- chave: frango; pectoralis major; resfriamento.
\end{abstract}

\section{SUMMARY}

PRE-CHILLING AND CHILLING TREATMENTS ON POULTRY BREAST MEAT QUALITY. A total of 402 poultry was processed in a commercial poultry processing plant and submitted to six chilling treatments. Initially, the carcasses were chilled by immersion in water and ice, followed by cooling at $-35^{\circ} \mathrm{C}$ or storage at $4^{\circ} \mathrm{C}$ for 20 hours. The treatments were: $\mathrm{A}\left(0^{\circ} \mathrm{C} / 30 \mathrm{~min},-35^{\circ} \mathrm{C} / 3 \mathrm{~h}\right.$ and $\left.15 \mathrm{~min}\right), \mathrm{B}\left(10^{\circ} \mathrm{C} / 30 \mathrm{~min}\right.$, $0^{\circ} \mathrm{C} / 30 \mathrm{~min},-35^{\circ} \mathrm{C} / 2 \mathrm{~h}$ and $\left.45 \mathrm{~min}\right), \mathrm{C}\left(10^{\circ} \mathrm{C} / 30 \mathrm{~min},-35^{\circ} \mathrm{C} / 3 \mathrm{~h}\right.$ and $\left.15 \mathrm{~min}\right), \mathrm{D}\left(20^{\circ} \mathrm{C} / 30 \mathrm{~min}, 0^{\circ} \mathrm{C} / 30 \mathrm{~min},-35^{\circ} \mathrm{C} / 2 \mathrm{~h}\right.$ and $\left.45 \mathrm{~min}\right), \mathrm{E}\left(20^{\circ} \mathrm{C} /\right.$ $30 \mathrm{~min},-35^{\circ} \mathrm{C} / 3 \mathrm{~h}$ and $\left.15 \mathrm{~min}\right)$ and $\mathrm{F}\left(20^{\circ} \mathrm{C} / 30 \mathrm{~min}, 0^{\circ} \mathrm{C} / 3 \mathrm{~h}\right.$ and $\left.15 \mathrm{~min}\right)$. Low temperatures used after evisceration, accelerated the onset and resolution of rigor in pectoralis major (PM) muscles. Up to 45 minutes post mortem, carcasses without pre-chilling (A) or pre-chilled at $10^{\circ} \mathrm{C}(\mathrm{B})$, showed lower $(\mathrm{P}<0.001) \mathrm{pH}$ values of 5.75 and 5.81 , while in carcasses pre-chilled at $20^{\circ} \mathrm{C}(\mathrm{D})$, the values were higher, reaching 5.95. After $4 \mathrm{~h}$ post mortem, the $\mathrm{R}$ values found in treatments $\mathrm{A}$ and $\mathrm{B}$, with averages of 1.51 and 1.44 , were higher $(\mathrm{P}<0.05)$ than the value of 1.32 found in treatment $\mathrm{D}$. The luminescence $\left(\mathrm{L}^{*}\right)$ was influenced $(\mathrm{P}<0.001)$ by the treatments (in treatments $\mathrm{A}, \mathrm{B}$ and $\mathrm{C}$, the averages were $48.2,47.7$ and 47.6 , while in treatments $\mathrm{D}$ and $\mathrm{E}$, they were 45.5 and 45.7 , respectively). The greater values for lightness coincided with treatments causing rapid rigor development in the PM muscle. The cooking loss and shear value were not affected by the treatments.

Keywords: chicken; chilling; pectoralis major

\section{1 - INTRODUÇÃO}

O resfriamento de carcaças de frango em baixas temperaturas tem sido descrito como indutor do encurtamento das fibras musculares pelo frio (que acarreta na redução da maciez, percebido após o cozimento), embora fibras brancas (maior percentual em frangos) sejam menos sensiveis a esse encurtamento. Em carcaças inteiras submetidas logo após o abate à $0^{\circ} \mathrm{C} / 80 \mathrm{~min}$ ou a $-12^{\circ} \mathrm{C} / 50 \mathrm{~min}$, DUNN, KILPATRICK \& GAULT [4] descreveram que naquelas resfriadas rapidamente houve variação para os resultados de força de cisalhamento (FC) nos músculos peitorais. Na análise de correlação, os autores encontraram coeficiente elevado $(r=-0,89)$ entre $\mathrm{pH}$ aos $15 \mathrm{~min}$ post mortem (p.m.) e comprimento de sarcômero. Isso demonstrou que a $-12^{\circ} \mathrm{C}$, aves com

\footnotetext{
Recebido para publicação em 28/06/2002. Aceito para publicação em 12/05/2004 (000918).

2. Universidade Federal de Lavras - Departamento de Ciências dos Alimentos. Caixa Postal 37 - Lavras-MG - CEP: 37200-000. E-mail: bressan@ufla.br

3. ITAL - Instituto de Tecnologia de Alimentos. Av. Brasil, 2880. Jd. Chapadão, Campinas-SP.

* A quem a correspondência deve ser enviada.
}

maior energia disponivel no pectoralis major (PM) aos $15 \mathrm{~min}$ p.m., sofreram maior encurtamento. PAPA \& FLETCHER [14] verificaram a susceptibilidade do PM de frango ao encurtamento p.m. e observaram que houve redução na retenção de água e na maciez, quando as amostras foram submetidas a cocção e ao processamento. LOCKER \& HAGYARD [12] descreveram que músculos acometidos de cold shortening resultaram em endurecimento da carne bovina após o cozimento. As alterações na maciez, facilmente perceptiveis, são consideradas como um dos maiores problemas associados ao abate comercial de frangos.

O objetivo deste trabalho foi verificar o efeito de diferentes temperaturas de pré-resfriamento e resfriamento de frangos sobre a velocidade de instalaçao do rigor mortis, maciez e perda de peso por cozimento do músculo PM.

\section{2 - MATERIAIS E MÉTODO}

O presente experimento foi conduzido em frigorífico comercial no município de Jundiaí-SP, no período de março a julho de 1997, compreendendo estações do outono e inverno cujas temperaturas médias variam de 18 a $25^{\circ} \mathrm{C}$ 


\section{1 - Caracterização dos animais}

Os frangos foram da linhagem Hubbard/Petterson, com 50 a 53 dias, peso médio de 1,8 a $2,5 \mathrm{~kg}$, sexo misto, criados em granjas comerciais. As aves, na granja foram preparadas para o abate de forma convencional, com jejum e dieta hídrica de $6 \mathrm{~h}$ antes da apanha. A apanha foi efetuada por equipe treinada e em cada gaiola foram colocados de 8 a 10 frangos. As aves foram transportadas da granja ao frigorífico por tempo inferior a $35 \mathrm{~min}$, em caminhões com carroceria adaptada, com velocidade média de $60 \mathrm{~km} / \mathrm{h}$.

$\mathrm{Na}$ indústria, as aves foram mantidas, no pré-abate, em instalações convencionais dotadas de umidificador de ar e ventiladores, por um periodo de 3 a 5 horas préabate. As aves não foram submetidas, enquanto na carga do caminhão a tratamento com água (chuveiro). Na plataforma de recepção, as aves foram penduradas pelos pés aos ganchos da nória de transporte e insensibilizadas por eletronarcose $(70 \mathrm{~mA}, 60 \mathrm{~V}$ e $1000 \mathrm{~Hz}$, por 10s). Após a insensibilização, as aves foram sangradas manualmente. A escaldagem foi realizada em água a $58^{\circ} \mathrm{C} / 2,5 \mathrm{~min}$, seguida da evisceração mecânica.

\section{2 - Tratamentos de pré-resfriamento e resfriamen- to}

As carcaças, após a evisceração, foram retiradas da linha e submetidas a 6 tratamentos de pré-resfriamento (PR) e resfriamento (R), aplicados no período de $15 \mathrm{~min}$ a $4 \mathrm{~h}$ p.m.:

$\mathrm{A}$ - Sem PR, onde as carcaças foram $\mathrm{R}$ a $0^{\circ} \mathrm{C} / 30 \mathrm{~min}$ e submetidas a $-35^{\circ} \mathrm{C} / 3 \mathrm{~h}$ e $15 \mathrm{~min}$.

$\mathrm{B}$ - PR a $10^{\circ} \mathrm{C} / 30 \mathrm{~min}, \mathrm{R}$ a $0^{\circ} \mathrm{C} / 30 \mathrm{~min}$, seguido de $\mathrm{R}$ a temperatura de $-35^{\circ} \mathrm{C} / 2 \mathrm{~h}$ e $45 \mathrm{~min}$.

$\mathrm{C}-\mathrm{PR}$ a $10^{\circ} \mathrm{C} / 30 \mathrm{~min}$, seguido de $\mathrm{R}$ a temperatura de $-30^{\circ} \mathrm{C} / 3 \mathrm{~h}$ e $15 \mathrm{~min}$.

$\mathrm{D}$ - PR a $20^{\circ} \mathrm{C} / 30 \mathrm{~min}, \mathrm{R}$ a $0^{\circ} \mathrm{C} / 30 \mathrm{~min}$, seguido de $\mathrm{R}$ a temperaturas de $-35^{\circ} \mathrm{C} / 2 \mathrm{~h}$ e $45 \mathrm{~min}$.

$\mathrm{E}-\mathrm{PR}$ a $20^{\circ} \mathrm{C} / 30 \mathrm{~min}$, seguido de $\mathrm{R}$ a temperatura de $-35^{\circ} \mathrm{C} / 3 \mathrm{~h}$ e $15 \mathrm{~min}$.

$\mathrm{F}-\mathrm{PR}$ a $20^{\circ} \mathrm{C} / 30 \mathrm{~min}$, seguido de $\mathrm{R}$ a $0^{\circ} \mathrm{C} / 3 \mathrm{~h}$ e $15 \mathrm{~min}$.

Os tratamentos foram aplicados por imersão das carcaças (30min) em cubas de PVC contendo soluções de água e gelo, cuja temperatura foi monitorada com termômetro (Corning M TMP-50). Durante a aplicação dos tratamentos, as carcaças foram revolvidas nas soluções para simular o "pré-chiller" e o "chiller" convencional.

\section{3 - Coleta, preparo de amostras e análises bio- químicas}

As amostras para as análises bioquímicas $(\mathrm{pH}$ e valor R), foram obtidas por 2 incisões paralelas na porção muscular do peito em sentido longitudinal ao externo (lado esquerdo e direito). À distância entre o externo, primeira e segunda linha de incisão foi de $1 \mathrm{~cm}$. Das duas amostras, retiradas na forma de tiras, foram separadas duas porções de 3,5g do músculo PM e con- geladas a $-196^{\circ} \mathrm{C}$. As amostras foram mantidas a $-196^{\circ} \mathrm{C}$ até o momento das análises. A determinação do valor $\mathrm{R}$ (em duplicata) foi realizada conforme HONIKEL \& FISCHER [7]. O pH foi determinado usando iodoacetato de sódio [1] e potenciômetro (Micronal M B375). As análises foram realizadas aos: 15; 45 e 75 minutos post mortem; e 4 e 24 horas post mortem, a fim de acompanhar a velocidade e instalação do rigor mortis em cada modalidade de tratamento.

\section{4 - Coleta, preparo de amostras e análises físicas}

Para as análises de perda de peso por cozimento (PPC) e força de cisalhamento (FC), as carcaças foram identificadas, embaladas, transportadas da indústria ao laboratório em caixas de polietileno expandido contendo gelo e armazenadas a $4^{\circ} \mathrm{C}$. Os músculos pectoralis superficialis (PS) e PM, esquerdo e direito, foram desossados às $24 \mathrm{~h}$ e usados para a determinação de PPC e FC.

A cor foi medida com colorimetro (Minolta Meter, modelo CR-200b) pelo Sistema CIELAB $\left(\mathrm{L}^{*}=\right.$ teor de luminosidade, $\mathrm{a}^{*}=$ teor de vermelho, $\mathrm{e} \mathrm{b}^{*}=$ teor de amarelo). As leituras foram tomadas no peito não desossado (pontos cranial, medial e distal do músculo PM), lado esquerdo e direito. A média de 6 leituras foi usada para cada carcaça.

Para a PPC, as amostras foram pesadas em balança semi-analítica (Mettler M P1210), embaladas em papel alumínio e cozidas em chapa a $180^{\circ} \mathrm{C}$ até atingir a temperatura interna de $82-85^{\circ} \mathrm{C}$ [2]. A diferença entre peso inicial e final dos músculos PM e PS correspondeu a PPC por carcaça.

As amostras empregadas na determinação da PPC foram utilizadas para avaliar a maciez [6]. Para medir a FC, as amostras foram colocadas com as fibras orientadas no sentido perpendicular às lâminas do WarnerBratzler, acoplado ao Instron M 2318. Seis a oito amostras na forma de paralelepípedo foram usadas na FC de cada peito.

\section{5 - Delineamento experimental e análise estatís- tica}

A atribuição dos tratamentos às unidades experimentais foi de acordo com um delineamento inteiramente casualizado, sendo cada frango considerado uma unidade experimental. Os dados foram submetidos à análise da variância e teste de média Duncan. O programa estatístico empregado foi o Statistica [22].

\section{3 - RESULTADOS E DISCUSSÃO}

\section{1 - Considerações sobre o pH aos $15 \mathrm{~min}$ post mortem}

$\mathrm{O} \mathrm{pH}$ aos $15 \mathrm{~min}$ variou de 5,75 a 5,83. Em frangos abatidos convencionalmente são reportados aos $10 \mathrm{~min}$ $\mathrm{pH}$ entre 6,09 a 6,30 [11] e aos $15 \mathrm{~min}$ valores entre 6,00 a 6,10 [21]. Resultados de $\mathrm{pH}$ com variações entre 5,74 a 5,90 foram descritos $1 \mathrm{~h}$ [20]. Entretanto, DUNN, 
KILPATRICS \& GAULT [5] obtiveram resultados elevados aos 30min $(6,70$ a 7,01$)$ e aos $90 \min (6,16$ a 6,74$)$. Confrontando os dados do presente trabalho com os desses autores, constata-se que o $\mathrm{pH}$ aos $15 \mathrm{~min}$ foi equivalente aos valores relatados aos $60 \mathrm{~min}$ post mortem.

Analisando-se a distribuição das classes de peito conforme a queda de $\mathrm{pH}$ no músculo PM (15min), observa-se que dos 45 peitos analisados, $11(24,4 \%)$ apresentaram valores abaixo de 5,7 , vinte e três $(51,1 \%) \mathrm{pH}$ entre 5,7 a 5,9 e onze $(24,4 \%)$ valores entre 5,9 a 6,1 . Portanto, 75,5\% das amostras apresentaram $\mathrm{pH} \leq 5,9$. Estes resultados discordam dos relatos de RISTIC [16] que encontrou $74,5 \%$ das amostras com valores entre 5,7 a 6,3 aos $15 \mathrm{~min}$. Considerando o valor de $\mathrm{pH}$ de 5,9 como o início do rigor [5], a maioria das carcaças avaliadas apresentaram no músculo PM instalação do rigor aos 15min. SHRIMPTON \& MILLER [19], descreveram que a instalação do rigor pode ocorrer aos 10min em condições de estresse antes do abate. SEEMANN [18] reportou, em músculos PM aos $35 \mathrm{~min}$, valores de $\mathrm{pH}$ baixo $(5,71)$, médio $(6,12)$ e alto $(6,39)$, sugerindo que $75,5 \%$ dos animais apresentaram rápido desenvolvimento da glicólise. Uma das possíveis explicações para a rápida instalação do rigor no presente trabalho estaria relacionado à linhagem comercial associada ao manejo pré-abate dispensado aos animais.

Os valores de $\mathrm{pH}$ aos $45 \mathrm{~min}$ variaram de 5,75 a 5,95. Em 45 peitos estudados $29(64,44 \%)$ mostraram $\mathrm{pH} \leq 5,9$, enquanto $16(35,55 \%)$ tiveram $\mathrm{pH}>5,9$. Às $4 \mathrm{~h}$ p.m., 95, 18\% dos músculos $\mathrm{PM}$ tinham $\mathrm{pH} \leq 5,9$. Esses resultados de $\mathrm{pH}$ demonstraram que, nas condições deste trabalho, entre 64,44 a $75,5 \%$ das carcaças apresentaram rápida glicólise. Por outro lado, 24,5 a $33,5 \%$ de carcaças evidenciaram lenta glicólise com pH de 5,9 aos 75 min post mortem.

Avaliando o desenvolvimento bioquímico, é esperado que o $\mathrm{pH}$ do músculo diminua ao longo do tempo (acúmulo de ácido lático). Em contrapartida, a quebra do ATP e da creatina fosfato provocam aumento de inosina e redução de adenosina no desenvolvimento do rigor, resultando em aumento no valor $\mathrm{R}$ [7].

\section{2 - Efeito dos tratamentos sobre as reações bio- químicas post mortem}

Os músculos PM das aves mostraram características bioquímicas e de cor semelhantes antes de receberem os tratamentos aos $15 \mathrm{~min}$ p.m. (Tabelas 1 e 2).

Os valores de $\mathrm{pH}(45 \mathrm{~min})$ foram afetados $(\mathrm{P}<0,001)$ pelos tratamentos de PR. Tratamentos A $\left(0^{\circ} \mathrm{C}\right)$ e $\mathrm{B}\left(10^{\circ} \mathrm{C}\right)$ mostraram valores de $\mathrm{pH}$ mais baixos $(5,75$ e 5,81 , respectivamente) que o tratamento $\mathrm{D}\left(20^{\circ} \mathrm{C}\right)(5,95)$. Esses resultados mostraram que a velocidade na instalação do rigor foi mais rápida em peitos de carcaças submetidas dos 15 aos $45 \mathrm{~min}$ p.m. em temperaturas de 0 e $10^{\circ} \mathrm{C}$, do que a $20^{\circ} \mathrm{C}$. Em carnes vermelhas, LOCKER \& HAGYARD [12] descreveram que carnes no pre rigor submetidas a temperaturas baixas $\left(16^{\circ} \mathrm{C}\right)$ apresentam reações bioquímicas que são aceleradas e que provocam redução na maciez, embora temperaturas elevadas pos- sam acelerar o rigor e, ocasionar o encurtamento por rigor que também desencadeia redução na maciez [4]. SMOLINSKA \& ABDUL-HALIM [20] descreveram que carcaças resfriadas rapidamente (valor $\mathrm{R}$ de 1,26 aos 40min, carcaças resfriadas sem pré-chiller com $28^{\circ} \mathrm{C}$ no interior do peito aos $15 \mathrm{~min}$ p.m.) apresentaram nos músculos peitorais maior velocidade na instalação do rigor do que carcaças resfriadas convencionalmente (valor $\mathrm{R}$ de 1,26 aos $65 \mathrm{~min}$, interior dos músculos a $8^{\circ} \mathrm{C}$ aos $\left.65 \mathrm{~min}\right)$.

TABELA 1. Médias para valor $\mathrm{R}$ e $\mathrm{pH}$ no músculo pectoralis major

\begin{tabular}{|c|c|c|c|c|c|c|c|c|c|c|c|}
\hline & \multirow{2}{*}{$\mathrm{N}$} & \multicolumn{2}{|c|}{$15 \mathrm{~min}$} & \multicolumn{2}{|c|}{$45 \mathrm{~min}$} & \multicolumn{2}{|c|}{$75 \mathrm{~min}$} & \multicolumn{2}{|c|}{$4 \mathrm{~h}$} & \multicolumn{2}{|c|}{$24 \mathrm{~h}$} \\
\hline & & $R$ value & $\mathrm{pH}$ & $R$ value & $\mathrm{pH}$ & $R$ value & $\mathrm{PH}$ & $R$ value & $\mathrm{pH}$ & $R$ value & $\mathrm{pH}$ \\
\hline A & 15 & $1,05^{\mathrm{a}}$ & $5,83^{a}$ & $1,17^{\mathrm{a}}$ & $5,75^{a}$ & - & - & $1,51^{\mathrm{a}}$ & $5,72^{a}$ & $1,37^{\mathrm{a}}$ & $5,70^{\circ}$ \\
\hline B & 15 & $1,04^{\mathrm{a}}$ & $5,75^{a}$ & $1,23^{\mathrm{ab}}$ & $5,81^{a}$ & $1,23^{a}$ & $5,75^{a}$ & $1,44^{\mathrm{ab}}$ & $5,75^{a}$ & $1,33^{a}$ & $5,68^{b}$ \\
\hline C & 15 & - & - & - & - & - & - & $1,31^{b}$ & $5,74^{a}$ & $1,35^{\mathrm{a}}$ & $5,72^{b}$ \\
\hline D & 15 & $1,11^{a}$ & $5,80^{a}$ & $1,28^{b}$ & $5,95^{\mathrm{b}}$ & $1,17^{\mathrm{a}}$ & $5,85^{b}$ & $1,32^{b}$ & $5,74^{\mathrm{a}}$ & $1,34^{\mathrm{a}}$ & $5,78^{a}$ \\
\hline E & 15 & - & - & - & - & - & - & $1,43^{\mathrm{ab}}$ & $5,67^{a}$ & $1,31^{a}$ & $5,83^{\mathrm{a}}$ \\
\hline $\mathrm{F}$ & 10 & - & - & - & - & - & - & $1,30^{b}$ & $5,69^{a}$ & $1,27^{\mathrm{a}}$ & $5,66^{b}$ \\
\hline $\mathrm{N}$ & & 45 & & 45 & & 30 & & 8 & & 8 & \\
\hline
\end{tabular}

$\mathrm{n}=$ número de unidades experimentais para as análise aos 15,45 e $75 \mathrm{~min}, 4$ e $24 \mathrm{~h}$ (15 ou 10 peitos, lado esquerdo e direito foram utilizados em cada momento de determinação pois essas análises são destrutivas)

$\mathrm{A}=0^{\circ} \mathrm{C} / 30 \mathrm{~min} ;-35^{\circ} \mathrm{C} / 3 \mathrm{~h}$ e 15 minutos; $0^{\circ} \mathrm{C} / 20 \mathrm{~h}$
$\mathrm{~B}=10^{\circ} \mathrm{C} / 30 \mathrm{~min} ; 0^{\circ} \mathrm{C} / 30 \mathrm{~min} ;-35^{\circ} \mathrm{C} / 2 \mathrm{~h} \mathrm{e} 45 \mathrm{~min} ; 0^{\circ} \mathrm{C} / 20 \mathrm{~h}$
$\mathrm{C}=10^{\circ} \mathrm{C} / 30 \mathrm{~min} ;-35^{\circ} \mathrm{C} / 3 \mathrm{~h}$ e $15 \mathrm{~min} ; 0^{\circ} \mathrm{C} / 20 \mathrm{~h}$

$\mathrm{C}=10^{\circ} \mathrm{C} / 30 \mathrm{~min} ;-35^{\circ} \mathrm{C} / 3 \mathrm{~h}$ e $15 \mathrm{~min} ; 0^{\circ} \mathrm{C} / 20 \mathrm{~h}$; $00^{\circ} \mathrm{C} / 2 \mathrm{hh}$

$\mathrm{D}=20^{\circ} \mathrm{C} / 30 \mathrm{~min} ; 0^{\circ} \mathrm{C} / 30 \mathrm{~min} ;-35^{\circ} \mathrm{C} / 2 \mathrm{~h}$ e $45 \mathrm{~min} ;$
$\mathrm{E}=20^{\circ} \mathrm{C} / 30 \mathrm{~min} ;-35^{\circ} \mathrm{C} / 3 \mathrm{~h} \mathrm{e} 15 \mathrm{~min} ; 0^{\circ} \mathrm{C} / 20 \mathrm{~h}$

$\mathrm{F}=20^{\circ} \mathrm{C} / 30 \mathrm{~min} ; 0^{\circ} \mathrm{C} / 23 \mathrm{~h}$ e $15 \mathrm{~min}$

a e bédias com expoentes diferentes, na coluna, são significativamente diferentes, teste de Duncan $(\alpha=0,05)$

Aos $45 \mathrm{~min}$, o valor $\mathrm{R}$ foi influenciado $(\mathrm{P}<0,05)$ pelos tratamentos. Carcaças submetidas a $20^{\circ} \mathrm{C}$ apresentaram valor $\mathrm{R}(1,28)$ mais elevado que aquelas tratadas a 0 e $10^{\circ} \mathrm{C}(1,17$ e 1,23 , respectivamente). Contrariando os resultados de $\mathrm{pH}$, isso demonstrou que carcaças submetidas dos 15 aos $45 \mathrm{~min}$ a $20^{\circ} \mathrm{C}$ apresentaram maior velocidade no consumo de ATP. Segundo HONIKEL \& FISCHER [7], músculos com valor $\mathrm{R}$ abaixo de 1,05 a quantidade de ATP é predominante, e acima de 1,05, a quantidade de IMP no meio é maior. Mais tarde, HONIKEL et al. [8] reportaram que valor $\mathrm{R}$ igual a 1,10 havia coincidido com a perda da extensibilidade muscular de bovinos e, portanto, este valor poderia ser utilizado como critério indicativo do rigor. Em frangos, o valor $\mathrm{R}$ que corresponde ao rigor é ponto de controvérsia entre autores. KHAN \& NAKAMURA [11] relataram que valor $\mathrm{R}$ de 1,0 foi encontrado em amostras em rigor. Entretanto SAMS \& MILLS [17] encontraram a $1 \mathrm{~h}$ p.m. valor R que variou de 1,09 a 1,12 em amostras com evidências de rigor.

Considerando os índices para valor $\mathrm{R}$ em rigor descritos por KHAN \& NAKAMURA [11] e HONIKEL \& FISCHER [7], os músculos deste trabalho estavam em rigor antes da aplicação dos tratamentos. KHAN [10] citou que temperaturas de $10,15,25$ e $37^{\circ} \mathrm{C}$ aplicadas as carcaças após a instalação do rigor não causaram efeitos sobre a maciez. Seguindo este raciocínio, os tratamentos aplicados após os 15min não afetariam a glicólise nos músculos PM utilizados neste trabalho. Entretanto, como os tratamentos influenciaram a instalação do rigor (pH 45 e 75min e valor R 45min), é pos- 
sível supor que os músculos aos 15 min estavam em pré-rigor, e portanto o valor $\mathrm{R}$ indicativo de rigor seria maior do que 1,1 conforme HONIKEL et al. [8] e SAMS \& MILLS [17].

Os músculos das aves $\mathrm{PR}$ a $10^{\circ} \mathrm{C}$ aos $75 \mathrm{~min}$ mostraram $\mathrm{pH}(5,75)$ significativamente $(\mathrm{P}<0,05)$ mais baixo, do que aves $\mathrm{PR}$ a $20^{\circ} \mathrm{C}(5,85)$, confirmando os resultados observados aos 45 minutos. Utilizando temperaturas entre 0 a $40^{\circ} \mathrm{C}$, DUNN, KILPATRICS \& GAULT [5] não observaram efeito sobre o pH quando os peitos foram incubados logo após o abate por 90 minutos. Entretanto, em períodos de incubação de até $6,33 \mathrm{~h}$ as amostras tratadas com 5 e $10^{\circ} \mathrm{C}$ apresentaram pH $(5,9)$ mais baixo, do que amostras mantidas a $30^{\circ} \mathrm{C}(\mathrm{pH}>6,0)$. Porém, SMOLINSKA, KOPEC \& POPIEL [21] descreveram que o rigor desenvolveu-se após $3 \mathrm{~h}$ p.m. em peitos de carcaças (pH de 5,5) submetidas ao R em água e gelo e, em 1,5h nas aves (pH de 5,9) não resfriadas.

O efeito do PR sobre a glicólise (valor R) pode ser observado comparando (4h) os tratamentos A, C e E que resultaram em valores $\mathrm{R}$ de 1,$51 ; 1,31$ e 1,43 , respectivamente. Entre os tratamentos de 0 e $10^{\circ} \mathrm{C}$, as médias foram diferentes $(\mathrm{P}<0,05)$ entre si, demonstrando que temperaturas de $0^{\circ} \mathrm{C}$ aceleraram o uso de ATP. Isso evidenciou que carcaças sem PR apresentaram resolução do rigor mais rápida no $\mathrm{PM}$, do que carcaças PR a 10 ou $20^{\circ} \mathrm{C}$. Esses resultados confirmaram o efeito das baixas temperaturas na aceleração da glicólise (45 e $75 \mathrm{~min}$ ). Efeito semelhante foi descrito em bovinos por HONIKEL \& HAMM [9] que observaram que temperaturas inferiores a $10^{\circ} \mathrm{C}$ aceleram as reações bioquímicas p.m., enquanto temperaturas acima de $10^{\circ} \mathrm{C}$ retardam as reações. Em bovinos, a aceleração das reações por temperaturas baixas (carcaças em pré-rigor) resulta em cold shortening [12]. Em frangos, BILGILI, EGBERT \& HOFFMAN [2], trabalhando com temperaturas de 41, 28, 14 e $0^{\circ} \mathrm{C}$, descreveram menor comprimento de sarcômero em peitos de carcaças submetidas a $0^{\circ} \mathrm{C}$. Resultados semelhantes foram reportados por PAPINAHO \& FLETCHER [15] que observaram maior encurtamento muscular em peitos desossados submetidos a $0^{\circ} \mathrm{C}$. Considerando os resultados de $\mathrm{pH}$ (45min) e de valor R (4h) encontrados no presente trabalho para os vários $R$, o tratamento A mostrou um efeito potencial para a ocorrência de encurtamento pelo frio e redução da maciez.

Os tratamentos de $\mathrm{PR}$ influenciaram $(\mathrm{P}<0,01)$ os valores de $\mathrm{pH}$ final. Comparando os $\mathrm{PR}$ (A, B e C), carcaças sem PR ou PR a $10^{\circ} \mathrm{C}(5,70 ; 5,68$ e 5,72 , respectivamente) foram diferentes $(\mathrm{P}<0,05)$ daquelas $\mathrm{PR}$ a $20^{\circ} \mathrm{C}$ (D e E) com médias de 5,78 e 5,83. Esses resultados mostraram que a ausência de PR ou PR a $10^{\circ} \mathrm{C}$ resultou em maior extensão da glicólise, do que o $\mathrm{PR}$ a $20^{\circ} \mathrm{C}$.

O efeito das temperaturas utilizadas após o PR pode ser observado quando comparado os tratamentos B e C com os tratamentos D a E. Observa-se que o $\mathrm{pH}$ às $4 \mathrm{~h}$ e o valor $\mathrm{R}$ às 4 e $24 \mathrm{~h}$ não foram afetados pelos tratamentos. Isso demonstrou que as temperaturas de resfriamento (água do chiller) de carcaças, aplicadas após 45min não influenciaram o rigor.
Utilizando temperaturas de $10,15,25$ e $37^{\circ} \mathrm{C}$ aplicadas a carcaças após a instalação do rigor, KHAN [10] não observou efeitos sobre a maciez. Como a textura de peito está associada com a atividade metabólica $p . m$., supõe-se que as diferentes temperaturas usadas por KHAN [10] não afetaram o desenvolvimento da fase final de rigor. De acordo com esses resultados, é possível que no presente trabalho, a ausência do efeito dos tratamentos de R (após os 45min) seja conseqüência da adiantada fase do rigor. Assim, infere-se que temperaturas baixas $\left(-35^{\circ} \mathrm{C}\right)$ aplicadas em carcaças de aves com rigor instalado tenham efeito semelhante aquele descrito para temperaturas entre 10 a $37^{\circ} \mathrm{C}$.

\section{3 - Considerações sobre o comportamento da lu- minosidade ( $\left.\mathrm{L}^{*}\right)$ no post mortem}

Analisando os dados para $\mathrm{L}^{*}$ cujas leituras foram realizadas entre os $15 \mathrm{~min}$ e às $24 \mathrm{~h}$ p.m., observou-se que houve uma evolução nos índices (aos 15min, as médias variaram de 40,4 a 41,3 , aos $75 \mathrm{~min}$ de 44,0 a 45,9 , às 4 h de 46,2 a 47,6 e às 24 h de 45,5 a 49,8) (Tabela 2). Estes dados mostram que as maiores modificações no teor de luminosidade ocorreram no período entre 15 a 75min. LYON \& CASSON [13] descreveram que ocorre uma evolução do L* entre 30 e $60 \mathrm{~min}$, resultado das reações bioquímicas p.m. nos músculos peitorais.

TABELA 2. Médias para os componentes de cor ( $\mathrm{L}^{*}, \mathrm{a}^{*} \mathrm{e} \mathrm{b}^{*}$ ) do pectoralis major

\begin{tabular}{|c|c|c|c|c|c|c|c|c|c|c|c|c|c|c|}
\hline \multirow{2}{*}{ T } & \multirow{2}{*}{ n } & \multicolumn{3}{|c|}{$15 \mathrm{~min}$} & \multicolumn{3}{|c|}{$45 \mathrm{~min}$} & \multicolumn{3}{|c|}{$75 \mathrm{~min}$} & \multicolumn{2}{|r|}{$4 \mathrm{~h}$} & \multicolumn{2}{|r|}{$24 \mathrm{~h}$} \\
\hline & & $L^{\star}$ & $a^{*}$ & $\mathrm{~b}^{*}$ & $L^{*}$ & $a^{*}$ & $b^{*}$ & $L^{*}$ & $a^{*}$ & $\mathrm{~b}^{*}$ & $L^{*}$ & $\begin{array}{ll}a^{*} & b^{*} \\
\end{array}$ & $L^{*}$ & $a^{*}$ \\
\hline & & $40,4^{a}$ & $2,52^{a}$ & $2,63^{a}$ & $45,1^{a}$ & $2,52^{a}$ & $4,16^{a}$ & - & & & $47,5^{a} 2$ & $2,01^{a} 5,27^{a b}$ & $48,2^{\mathrm{ab}}$ & $2,44^{a} 3,07^{a}$ \\
\hline B & 15 & $41,3^{\mathrm{a}}$ & $2,38^{\mathrm{a}}$ & $3,11^{\mathrm{a}}$ & $44,7^{\mathrm{a}}$ & $2,86^{a}$ & $4,16^{a}$ & $45,9^{a}$ & $2,75^{\mathrm{a}}$ & $4,30^{a}$ & $47,1^{a} 2$ & $2,79^{\mathrm{a}} 6,12^{\mathrm{a}}$ & $47,7^{\mathrm{abc}}$ & $2,70^{a} 3,45^{a}$ \\
\hline $\mathrm{C}$ & 15 & - & - & - & - & - & - & - & - & - & $46,8^{a} 2$ & $2,57^{\mathrm{a}} 3,95^{\mathrm{bc}}$ & $47,6^{\mathrm{bc}}$ & $2,44^{\mathrm{a}} 3,74^{\mathrm{a}}$ \\
\hline D & 15 & $41,1^{\mathrm{a}}$ & $1,79^{b}$ & $2,46^{a}$ & $42,2^{b}$ & $2,15^{a}$ & $3,32^{\mathrm{a}}$ & $44,6^{a}$ & $2,26^{\mathrm{b}}$ & $4,35^{a}$ & $46,9^{\mathrm{a}} 2$ & $2,25^{\mathrm{a}} 3,96^{\mathrm{bc}}$ & $45,5^{d}$ & $2,60^{\mathrm{a}} 3,11^{\mathrm{a}}$ \\
\hline E & 15 & - & - & - & - & - & - & - & - & - & $47,6^{a} 1$ & $1,64^{\mathrm{ab}} 2,84^{\mathrm{c}}$ & $45,7^{\text {cd }}$ & $2,67^{\mathrm{a}} 3,08^{\mathrm{a}}$ \\
\hline$F$ & 10 & - & - & - & - & - & - & - & - & - & $46,2^{\mathrm{a}} 0$ & $0,47^{b} 2,56^{c}$ & $49,8^{\mathrm{a}}$ & $2,29^{\mathrm{a}} 4,19^{\mathrm{a}}$ \\
\hline & & & 45 & & & 45 & & & 30 & & & 85 & & 85 \\
\hline
\end{tabular}

$\mathrm{A}=0^{\circ} \mathrm{C} / 30 \mathrm{~min} ;-35^{\circ} \mathrm{C} / 3 \mathrm{~h}$ e $15 \mathrm{~min} ; 0^{\circ} \mathrm{C} / 20 \mathrm{~h}$

$\mathrm{A}=0^{\circ} \mathrm{C} / 30 \mathrm{~min} ;-35^{\circ} \mathrm{C} / 3 \mathrm{~h} \mathrm{e} 15 \mathrm{~min} ; 0^{\circ} \mathrm{C} / 20 \mathrm{~h}$
$\mathrm{~B}=10^{\circ} \mathrm{C} / 30 \mathrm{~min} ; 0^{\circ} \mathrm{C} / 30 \mathrm{~min} ;-35^{\circ} \mathrm{C} / 2 \mathrm{~h} \mathrm{e} 45 \mathrm{~min} ; 0^{\circ} \mathrm{C} / 20 \mathrm{~h}$
$\mathrm{C}=10^{\circ} \mathrm{C} / 30 \mathrm{~min} ;-35^{\circ} \mathrm{C} / 3 \mathrm{~h} \mathrm{e} 15 \mathrm{~min} ; 0^{\circ} \mathrm{C} / 20 \mathrm{~h}$

$\mathrm{D}=20^{\circ} \mathrm{C} / 30 \mathrm{~min} ; 0^{\circ} \mathrm{C} / 30 \mathrm{~min} ;-35^{\circ} \mathrm{C} / 2 \mathrm{~h}$ e $45 \mathrm{~min} ; 0^{\circ} \mathrm{C} / 20 \mathrm{~h}$

$\mathrm{E}=20^{\circ} \mathrm{C} / 30 \mathrm{~min} ;-35^{\circ} \mathrm{C} / 3 \mathrm{~h}$ e $15 \mathrm{~min} ;$
$\mathrm{F}=20^{\circ} \mathrm{C} / 30 \mathrm{~min} ; 0^{\circ} \mathrm{C} / 23 \mathrm{~h} \mathrm{e} 15 \mathrm{~min}$

$\mathrm{n}=$ número de unidades experimentais para cada momento de análise

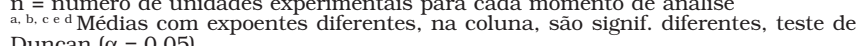
Duncan $(\alpha=0,05)$

$\mathrm{L}^{*}=$ luminosidade (preto $=0$ e branco $=100$ )

$\mathrm{a}^{*}=$ tendência para vermelho (verde $=-80$ e vermelho $=100$ )

$\mathrm{b}^{*}=$ tendência para $\mathrm{o}$ amarelo $(\mathrm{azul}=-50$ e amarelo $=70)$

\section{4 - Efeito dos tratamentos sobre os componen- tes da cor ( $L^{*}, a^{*}$ e $\left.b^{*}\right)$}

$O L^{*}$ foi influenciado $(\mathrm{P}<0,001)$ pelos tratamentos de $\mathrm{PR}$ aos $45 \mathrm{~min}$. Comparando os tratamentos A, B e D observa-se que peitos de carcaças sem PR ou PR a $10^{\circ} \mathrm{C}$ $(45,1,44,7$, respectivamente) mostraram maior luminosidade, do que aqueles $\mathrm{PR}$ a $20^{\circ} \mathrm{C}(42,2)$. Estes resultados demonstraram que a evolução da luminosidade em peito de frango foi mais elevada em carcaças sem $\mathrm{PR}$ ou PR a $10^{\circ} \mathrm{C}$. Considerando que o $\mathrm{L}^{*}$ manifeste as alterações bioquímicas, é possível inferir que carcaças sem $\mathrm{PR}$ ou $\mathrm{PR}$ a $10^{\circ} \mathrm{C}$ tiveram uma evolução mais rápida do que aquelas $\mathrm{PR}$ a $20^{\circ} \mathrm{C}$. Isso confirma os resultados de $\mathrm{pH}$ obtidos aos 45 e $75 \mathrm{~min}$ post mortem. 
A análise da variância determinou efeito $(\mathrm{P}<0,001)$ dos tratamentos de $\mathrm{R}$ sobre o $\mathrm{L}^{*}$ às $24 \mathrm{~h}$. Carcaças sem $\mathrm{PR}$ ou PR a $10^{\circ} \mathrm{C}(\mathrm{A}, \mathrm{B}$ e C) com médias de 48,$2 ; 47,7$ e 47,6 diferiram $(\mathrm{P}<0,05)$ daquelas carcaças $\mathrm{PR}$ a $20^{\circ} \mathrm{C}(\mathrm{D}$ e E) com médias de 45,5 e 45,7. Esses resultados evidenciam que as temperaturas utilizadas, logo após a evisceração, influenciaram o L* às $24 \mathrm{~h}$, onde temperaturas de 0 ou $10^{\circ} \mathrm{C}$ resultam em elevado teor de luminosidade. Porém, as temperaturas utilizadas após os 45 min do abate não afetaram o L*.

Os resultados das análises bioquímicas e cor foram submetidas a análise de correlação aos 15 e $45 \mathrm{~min}, 4$ e $24 \mathrm{~h}$ e foi observado que: a) aos $15 \mathrm{~min}$, o $\mathrm{L}^{*}$ foi positivamente correlacionado ao valor $\mathrm{R}$ (com $\mathrm{r}=0,53, \mathrm{P}=0,01 \%$ ), demonstrando que, quanto maior foi a hidrólise de ATP (maior valor R) maior foi a luminosidade; b) quando analisado a relação luminosidade e $\mathrm{pH}$, os índices de correlação foram inversos aos $45 \mathrm{~min}(\mathrm{r}=-0,65, \mathrm{P}=0,01 \%)$, às $4 \mathrm{~h}(\mathrm{r}=-0,51, \mathrm{P}=0,01 \%)$ e às $24 \mathrm{~h}(\mathrm{r}=-0,54, \mathrm{P}=0,01 \%)$, mostraram que, quanto mais baixo foram os valores de $\mathrm{pH}$ mais elevados foram os índices de luminosidade. Considerando essas correlações, é possivel associar a evolução da luminosidade com a evolução das reações de hidrólise de ATP (valor R) ou com a glicólise $(\mathrm{pH})$. Dessa forma, quanto mais rápida a instalação do rigor no PM maior é a luminosidade (às 24h, o índice de correlação indicou que a luminosidade também está associado a extensão de glicólise, ou seja, quanto menor é o pH final, maior é a luminosidade).

\section{5 - Efeito dos tratamentos sobre a perda de peso por cozimento (PPC) e força de cisalhamento (FC)}

A PPC não foi influenciada pelos tratamentos ( $\mathrm{Ta}$ bela 3). Esses resultados estão de acordo com DUNN, KILPATRICS \& GAULT [5] que, utilizando temperaturas de $0,5,10,20,30$ e $40^{\circ} \mathrm{C}$, aplicadas após o abate não observaram efeito da temperatura sobre a PPC. Por outro lado, esses resultados discordam de BILGILI, EGBERT \& HOFFMAN [2] que encontraram em carcaças submetidas a 41 e $28^{\circ} \mathrm{C}$ perdas superiores a $29 \%$, enquanto que para carcaças tratadas a 14 e $0^{\circ} \mathrm{C}$ as perdas foram de 27,9 e $25,1 \%$, respectivamente.

TABELA 3. Médias para PPC e FC no músculo pectoralis major

\begin{tabular}{c|ccccc}
\hline Tratamentos & N & PPC (\%) & n & FC (kgf/g) \\
\hline A & 17 & $28,44^{\mathrm{a}}$ & 17 & $2,92^{\mathrm{a}}$ \\
B & 19 & $29,03^{\mathrm{a}}$ & 19 & $3,04^{\mathrm{a}}$ \\
C & 18 & $28,08^{\mathrm{a}}$ & 18 & $3,22^{\mathrm{a}}$ \\
D & 21 & $28,11^{\mathrm{a}}$ & 21 & $3,50^{\mathrm{a}}$ \\
E & 20 & $28,90^{\mathrm{a}}$ & 20 & $3,34^{\mathrm{a}}$ \\
F & 17 & $28,36^{\mathrm{a}}$ & 17 & $3,24^{\mathrm{a}}$ \\
\hline N & \multicolumn{3}{c}{112} & \multicolumn{3}{c}{112}
\end{tabular}

$\mathrm{A}=0^{\circ} \mathrm{C} / 30 \mathrm{~min} ;-35^{\circ} \mathrm{C} / 3 \mathrm{~h}$ e 15 minutos; $0^{\circ} \mathrm{C}$ por $20 \mathrm{~h}$ $\mathrm{B}=10^{\circ} \mathrm{C} / 30 \mathrm{~min} ; 0^{\circ} \mathrm{C} / 30 \mathrm{~min} ;-35^{\circ} \mathrm{C} / 2 \mathrm{~h}$ e $45 \mathrm{~min} ; 0^{\circ} \mathrm{C} / 20 \mathrm{~h}$ $\mathrm{C}=10^{\circ} \mathrm{C} / 30 \mathrm{~min} ;-35^{\circ} \mathrm{C} / 3 \mathrm{~h}$ e $15 \mathrm{~min} ; 0^{\circ} \mathrm{C} / 20 \mathrm{~h}$ $\mathrm{D}=20^{\circ} \mathrm{C} / 30 \mathrm{~min} ; 0^{\circ} \mathrm{C} / 30 \mathrm{~min} ;-35^{\circ} \mathrm{C} / 2 \mathrm{~h}$ e $45 \mathrm{~min} ; 0^{\circ} \mathrm{C} / 20 \mathrm{~h}$ $\mathrm{E}=20^{\circ} \mathrm{C} / 30 \mathrm{~min} ;-35^{\circ} \mathrm{C} / 3 \mathrm{~h}$ e $15 \mathrm{~min} ; 0^{\circ} \mathrm{C} / 20 \mathrm{~h}$ $\mathrm{F}=20^{\circ} \mathrm{C} / 30 \mathrm{~min} ; 0^{\circ} \mathrm{C} / 23 \mathrm{~h}$ e $15 \mathrm{~min}$ $\mathrm{n}=$ número de unidades experimentais b. c ed Médias com expoentes diferentes, na coluna, são signif. diferentes, teste de Duncan $(\alpha=0,05)$
A FC não foi afetada pelos tratamentos. Esses resultados demonstraram que os tratamentos de PR ou $\mathrm{R}$, não afetaram a maciez dos músculos PM. As médias de FC variaram de 2,92 a 3,50kgf/g. Esses resultados ficaram abaixo de 5,5 a $5,8 \mathrm{kgf} / \mathrm{g}$ descritos para peitos de frango desossados convencionalmente [3]. Isso evidencia que a maciez encontrada foi elevada, embora nas amostras do tratamento A tenha ocorrido rápida glicólise (condição bioquímica potencial para o "encurtamento pelo frio").

\section{4 - CONCLUSÕES}

A velocidade na instalação e resolução do rigor foi acelerada em peitos de carcaças sem pré-resfriamento submetidas a $0^{\circ} \mathrm{C} / 30 \mathrm{~min}$, seguida de resfriamento a $-35^{\circ} \mathrm{C}$ por $3 \mathrm{~h}$ e 15 minutos. As temperaturas de resfriamento, utilizadas após $45 \mathrm{~min}$ do abate, não influenciaram a velocidade das reações químicas post mortem. A perda de peso por cozimento e a força de cisalhamento não foram afetadas pelas condições de pré-resfriamento. Nas operações de obtenção de carcaças inteiras, a temperatura de pré-resfriamento pode ser reduzida de $20^{\circ} \mathrm{C}$ para $10^{\circ} \mathrm{C}$ ou a etapa de pré-resfriamento pode ser eliminada sem prejuízos da cor, da perda de peso por cozimento e da maciez de peitos de frangos.

\section{5 - REFERÊNCIAS BIBLIOGRÁFICAS}

[1] BENDALL, J.R. "Post mortem" changes in muscle. Relation between muscle $\mathrm{pH}$ and important biochemical parameters during the post mortem changes in mammalian muscles. In: The structure and function of muscle. New York, Academic Press. v. 2, p. 143-157, 1973.

[2] BILGILI, S.F.; EGBERT, W.R.; HOFFMAN, D.L. Research note: Effect of post mortem ageing temperature on sarcomere lengh and tenderness of broiler pectoralis major. Poultry Science. Champaign, v. 68, n. 11, p. 1588-1591, 1989.

[3] CONTRERAS, C.J.C. Efeitos do atordoamento elétrico, estimulação elétrica e da desossa à quente na qualidade da carne do peito de frango "Pectoralis major". Campinas, 1995. 150p. Tese - Faculdade de Engenharia de Alimentos, Universidade Estadual de Campinas.

[4] DUNN, A.A.; KILPATRICK, D.J.; GAUlT, N.F.S. Contribution of "rigor shortening" and "cold shortening" to variability in the texture of pectoralis major muscle from commercially processed broilers. British Poultry Science. Langford, v. 36, n. 3, p. 401-413, 1995.

[5] DUNN, A.A.; KILPATRICS, D.J.; GAULT, N.F.S. Effect of post mortem temperature on chicken pectoralis major: muscle shortening and cooked meat tenderness. British Poultry Science. Langford, v. 34, n. 4, p. 689-697, 1993.

[6] FRONING, G.W.; BABJI, A.S.; MATHER, F.B. The effect of preslauther temperature, stress, struggle and anesthetization on colour and textural characteristics of turkey muscle. Poultry Science. Champaign, v. 57, n. 3, p. 630-633, 1978.

[7] HONIKEL, K.O.; FISCHER, C.A. A rapid method for the detection of PSE and DFD porcine muscles. Journal of Food Science. Chicago, v. 42, n. 7, p. 1663-1676, 1977. 
[8] HONIKEL, K.O.; FISCHER, C.; HAMID, A.; HAMM, R. Influence of post mortem changes in bovine muscle on the water-holding capacity of beef. Post mortem storage of muscle at $20^{\circ} \mathrm{C}$. Journal of Food Science. Chicago, v. 46, n. 1, p. $1-7,1981$.

[9] HONIKEL, K.O.; HAMM, R. La influencia del refrigerado sobre las cualidades de la carne vacuna recién faenada. Fleischwirts, Español. Frankfurt, n. 2, p. 16-22, 1980.

[10] KHAN, A.W. Effect of temperature during post mortem glycolysis and dephosphorylation of high energy phosphates on poultry meat tenderness. Journal of Food Science. Chicago, v. 36, n. 1, p. 120-1, 1971.

[11] KHAN, A.W.; NAKAMURA, R. Effects of pre-and post mortem glycolysis on poultry tenderness. Journal of Food Science. Chicago, v. 35, n. 3, p. 266-267, 1970.

[12] LOCKER, R.H.; HAGYARD, C.J.A. Cold shortening effect in beef muscle. Journal of the Science Food Agriculture. Wageningen, v. 14, p. 787-793, 1963

[13] LYON, C.E.; CASSON, J.A. Effect of water chilling on objective color of bruised and unbruised broiler tissue. Poultry Science. Champaign, v. 74 , n. 11 , p. 1894-1899, 1995.

[14] PAPA, C.M.; FLETCHER, D.L. Pectoralis muscle shortening and rigor development at different locations within the broiler breast. Poultry Science. Champaign, v. 67, n. 4 , p. 635-640, 1988.

[15] PAPINAHO, P.A.; FLETCHER, D.L. The influence of temperature on broiler beast muscle shortening and extensibility. Poultry Science. Champaign, v. 75, p. 797-802, 1996.

[16] RISTIC, M. The relation between duration of fattening, retail grade and meat quality of broilers. Fleischerei. Frankfurt, v. 30, n. 9, p. 696-698, 1979.
[17] SAMS, A.R.; MILLS, K.A. The effect of feed withdrawal duration on the responsiveness of broiler pectoralis to rigor mortis acceleration. Poultry Science. Champaign, v. 72, n. 9, p. 1789-1796, 1993.

[18] SEEMANN, G. Beziehungen zwischen der pH wert änderung nach dem schlachten und anderen fleischqualitätsparametern beim hähnchen. Fleischwirtschaft. Frankfurt, v. 66, n. 4, p. 604-606, 1986.

[19] SHRIMPTON, D.H.; MILLER, W.S. Some causes of toughness in broilers. II. Effects of breed, management and sex. Poultry Science. Champaign, v. 1, p. 111-116, 1960.

[20] SMOLINSKA, T.; ABDUL-HALIM, F. The effects of freezing systems and frozen storage on the quality of broiler tissue muscle. Archiv fuer Gefluegelkunde. Stuttgart, v. 56, n. 2, p. 80-85, 1992.

[21] SMOLINSKA, T.; KOPEC, W.; POPIEL, A. Effects of salt modified post mortem changes in chicken meat on sausages quality. In: EUROPEAN WPSA SYMPOSIUM ON THE OF POULTRY MEAT, IX $^{\text {th }} \cdot 22-25$ august, Ulmer, 1989. Procedings... Hohenheim: Scholtyssek University Hohenheim. 1989. p. 191-7.

[22] STATISTICA. Copyright (c) Stat Soft, Inc. Release 5.0. Tulsa. 1995.

\section{6 - AGRADECIMENTOS}

Os autores agradecem ao CNPq pela concessão de bolsa de produtividade em pesquisa e a CEVAL Alimentos, pelo empréstimo das instalações (Jundiaí) e doação de amostras. 